\title{
DIGITAL VIDEOMAGNETOGRAMS IN REAL TIME
}

\author{
THOMAS J. JANSSENS and NEAL K. BAKER \\ The Aerospace Corporation, San Fernando Observatory, Los Angeles, California, U.S.A.
}

\begin{abstract}
The Aerospace - NASA Videomagnetograph began operation one month ago, two years after components were ordered and construction began. The design grew out of a desire to obtain magnetic fields in real time using an optical filter. The aim was to study and analyze magnetic configurations and changes, quantitatively if possible, with high spatial and temporal resolution and as much sensitivity as possible. This instrument is restricted to the line-of-sight component of the magnetic field and is primarily intended for high resolution studies of selected regions of the sun. The rationale behind our approach is shown in the next section and the design details in the following.
\end{abstract}

\section{Analysis of An Idealized Magnetograph}

In most solar magnetographs the measurement of the line-of-sight component requires the same processes, namely: light collection, spectral isolation, polarization selection, detection, subtraction, scanning, and data presentation.

The performance characteristics include the sensitivity, $\Delta B$; the area of a resolution element, $a$; the time resolution, $T$; and the number of resolution elements per scan, $n$. If we assume a given magnetic line and noise determined solely by the photon limit then we find that the product $n(\Delta B)^{-2} T^{-1} a^{-1}$ is invariant such that if $n, T$ and $a$ are changed, $\Delta B$ varies to hold the product constant. In theory this allows any desired trade off between spatial resolution, temporal resolution, and field of view but in practice such versatility is rather limited in actual magnetographs. Thus this product can perhaps be used then as a kind of performance figure of merit. It is proportional to the rate of photon detection and depends on the product $\varepsilon A$ (or $\varepsilon A N$ for a multichannel detector) where $\varepsilon$ is the overall efficiency of the system, $A$ is the area of the objective lens, and $N$ is the effective number of data channels. The results are not surprising: that someone with an ideal detector would want a large aperture telescope, a high efficiency, and a multichannel detector.

Our approach has been to concentrate on increasing the effective number of parallel data channels, while using a rather small aperture. The number of data channels and mode of scanning are interrelated. Three types of scanning might be called a point-bypoint scan, a slit scan, and a simultaneous exposure. In the point-by-point scan light from one element passes through a spectrograph and is analysed with the procedure repeated for each element in turn. This gives an effective $N=1$. In a slit scanning system such as the photographic subtraction technique developed by Leighton, photons along the exit slit of a spectroheliograph are simultaneously detected. This typically gives an effective $N$ of 200 to 1000 . The third method of simultaneous exposure requires the detection of photons from the entire image at once. This could have an effective $N=40000$ for a $200 \times 200$ array.

Simultaneous exposure requires use of a filter for spectral isolation rather than a spectrograph. This generally restricts one's versatility in the narrowness of the spec- 
trum selected and the number of lines available for use, though filter technology has been steadily improving.

The two possible sensors for simultaneous exposure are film and video. With film one is restricted to a low duty cycle or a large expenditure of film. For example to take $0.2 S$ exposures each $10 S$ would decrease the effective $N$ by a factor of 50 while to take exposures much more frequently would consume an enormous amount of film. In addition, photographic subtraction can be time consuming, difficult, and suitable from only single or double cancellation. Certainly photographic subtraction would not yield real time results.

A television camera is not without difficulties either. They usually cannot match film in resolution and signal-to-noise ratio at present. Also, the target of a video camera can hold only a given number of photoelectrons before being read off, so if one is to maximize the rate of photon detection the camera must be scanned at a fast rate, not operated in a slow scan mode. In a fast scan mode, the rates and capacity necessary to store and subtract video images in real time are quite a problem.

We decided to store, subtract, and average (enhance) the video images digitally rather than by analog means but found that the usual method of digitizing and storing the data for later analysis does not work because the video data is 4 million words per second, about 100 times faster than the usual magnetic tape recorder. A thousand reels of magnetic tape would be filled in only one hour at video rates.

Computing with the data is much faster than storing it but for our task the required speed and storage capacity would require a very large and expensive general purpose computer which was not available. To solve the problem we designed our own special purpose computer which would operate at these high video rates. The heart of the video computer is a high speed adder with a memory unit consisting of a digital magnetic disc which is used with sequential addressing instead of random accessing. The disc has 72 tracks, 130000 bits per track and rotates at $30 \mathrm{~Hz}$. The advantages of a disc memory over core memory are, a much lower cost, a greater capacity, and higher access times. In addition, we can simultaneously use different parts of the disc for different tasks.

\section{Description of the Videomagnetograph}

The system shown in block diagram form in Figure 1 has four organization parts: (i) optical and video, (ii) data gathering and preprocessing section, (iii) display section, and (iv) analytic section.

The optical section consists of a telescope with a $15 \mathrm{~cm}$ diameter lens which forms a real image which is further enlarged onto the face of an SEC Vidicon. Spectral isolation of one wing of the $5324 \AA$ line of $\mathrm{Fe} I$ is achieved by a hybrid filter with a bandpass of $0.11 \AA$ similar to the filter developed by H. Ramsey of Lockheed. An electro optic crystal or a quarter wave plate is switched to alternately admit right and left handed circularly polarized light. One TV line is 1.5 arc s and the field of view is 300 arc s on a side. 
The data gathering and preprocessing section includes the analog-to-digital converter, high speed adder, and 32 tracks of the disc divided into bands 1 and 2.

The incoming video signal is digitized into 8 bits (256 levels) by a 4 megacycle converter which is synchronized with the data flow of the disk. The output of the analog-to-digital converter can be added to or subtracted from a previously stored array in the data gathering area of the disk and then stored, thereby erasing the previous image array. This operation is controlled by the disk interface controller

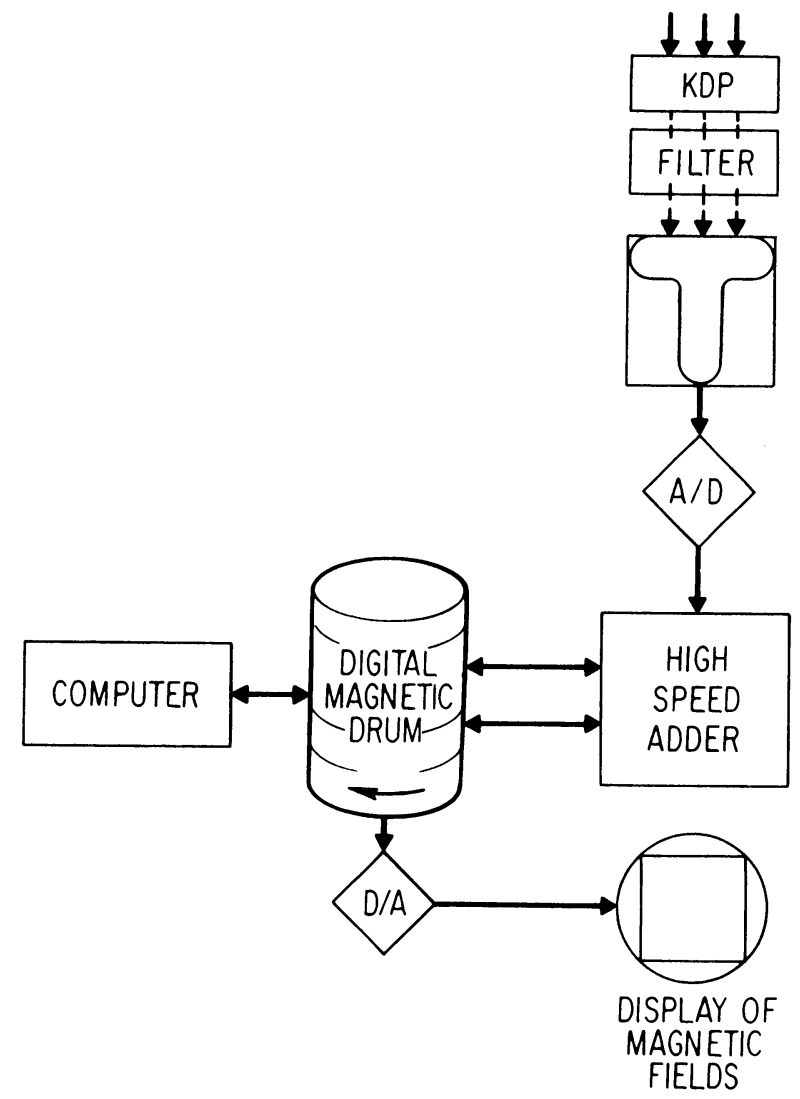

Fig. 1. Block diagram of the Aerospace - NASA Videomagnetograph.

which requires one instruction per frame whether to add or subtract the incoming image. It is operated on an interrupt basis by a small general purpose computer attached to the system.

After the completion of the data gathering mode, the system can be interrogated for information on the entire frame. At present, we can get the number of elements greater than a certain value, value of the $n$th largest element, and $m$ th smallest element, or the average value. The position of the element can not be found in this interrogation mode. 


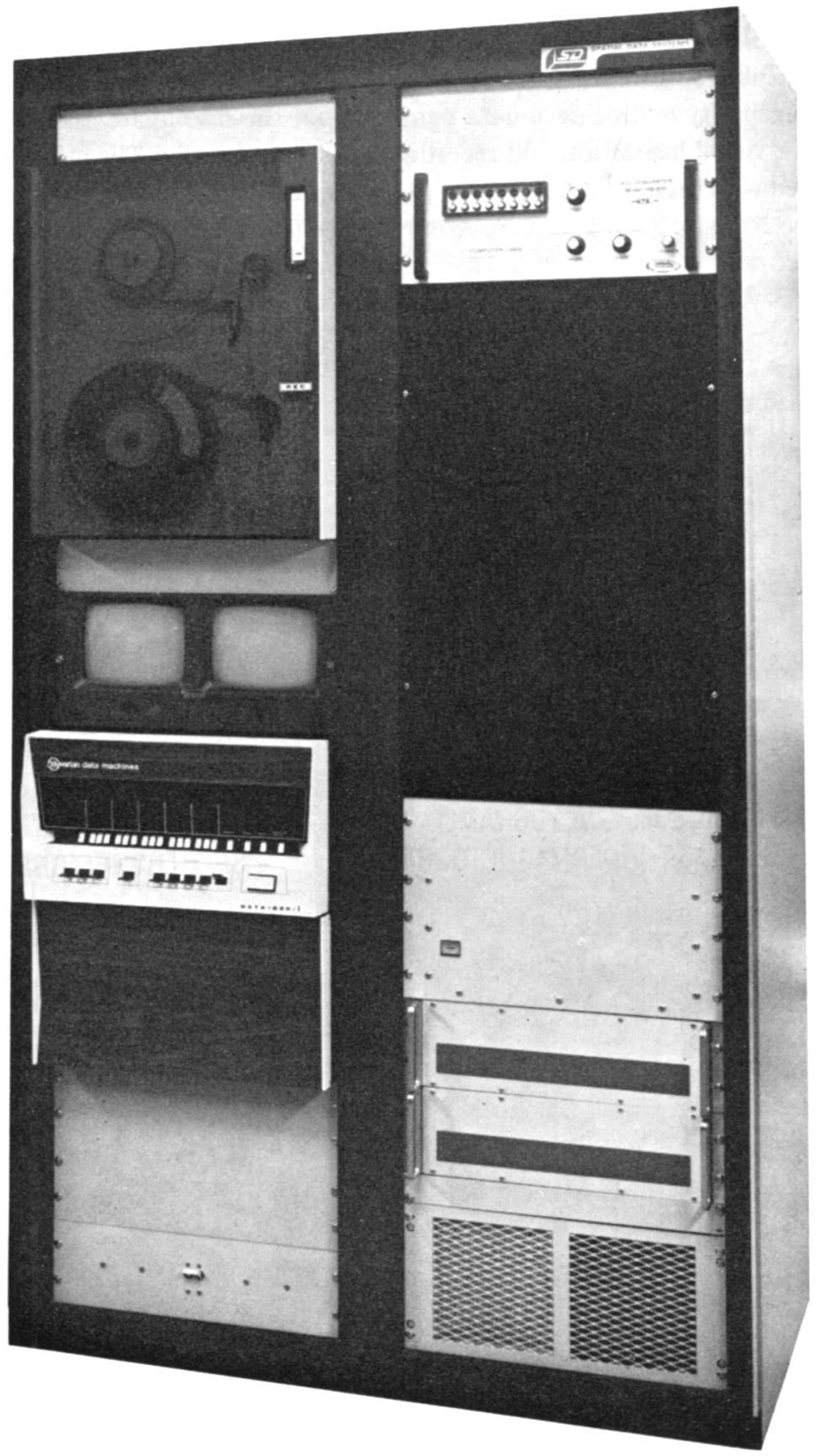

Fig. 2. Digital Processor for the Videomagnetograph. 
For instance, the time to obtain the $n$th largest element in the array of 100000 points is $0.5 \mathrm{~s}$. This information is mainly used to set the levels for the display and to make distribution plots.

In the display section after a number of magnetic frames have been averaged to enhance sensitivity and achieve data compression, the results are displayed on a TV monitor for visual inspection and recording photographically. The magnetic field can be displayed with each brightness made to correspond to any desired magnetic strength in a number of ways, ranging from arbitrary selection of 8 to 16 gray levels to automatic selection by the computer. The display can be either in a positive or negative mode, logarithmic or linear scaling. This display data is transferred to the display section of the disk and displayed to the operator. The 4 bit (16 gray levels) data is

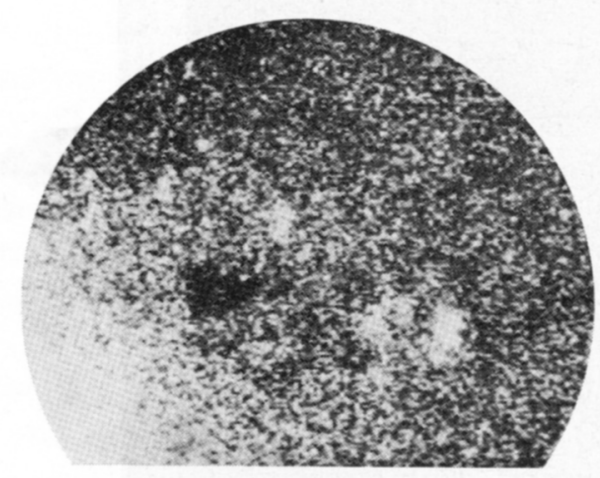

FIRST VIDEOMAGNETOGRAM BY AEROSPACE-NASA VIDEOMAGNETOGRAPH

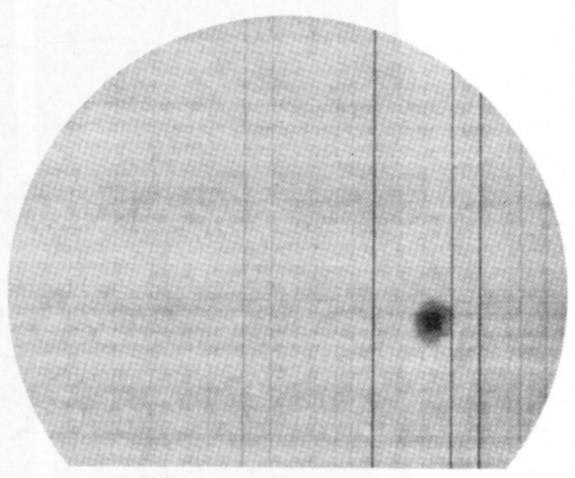

CONTINUUM SPECTROHELIOGRAM

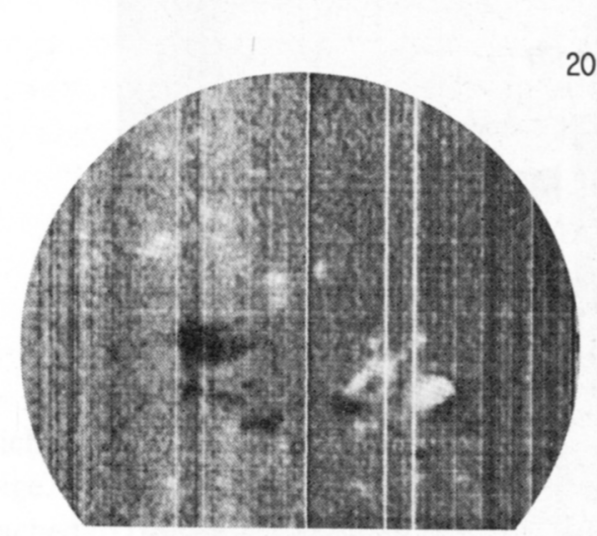

MAGNETIC FIELDS BY PHOTOGRAPHIC SUBTRACTION

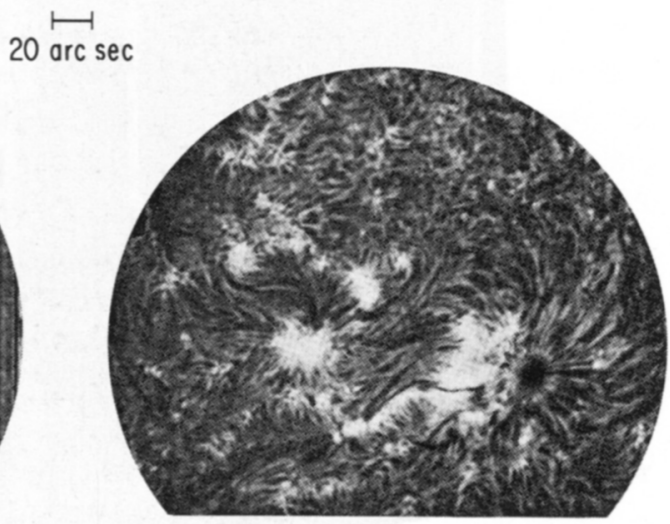

Ha FILTERGRAM

Fig. 3. First real time display of magnetic fields on July 17, 1970 at the Aerospace San Fernando Observatory. $40 \mathrm{~s}$ were required for 500 subtractions. Other pictures are for comparison and confirmation. 
connected to a digital-to-analog convertor and fed to two TV monitors which are synchronized to the disk. The operator can use a light pen on the monitor to select points or areas on which he wants more information, such as the numerical value of a specific point or position of the point. For areas, the operator can define rectangles or circles of various sizes in which he wants average values, maximum value, or minimum value.
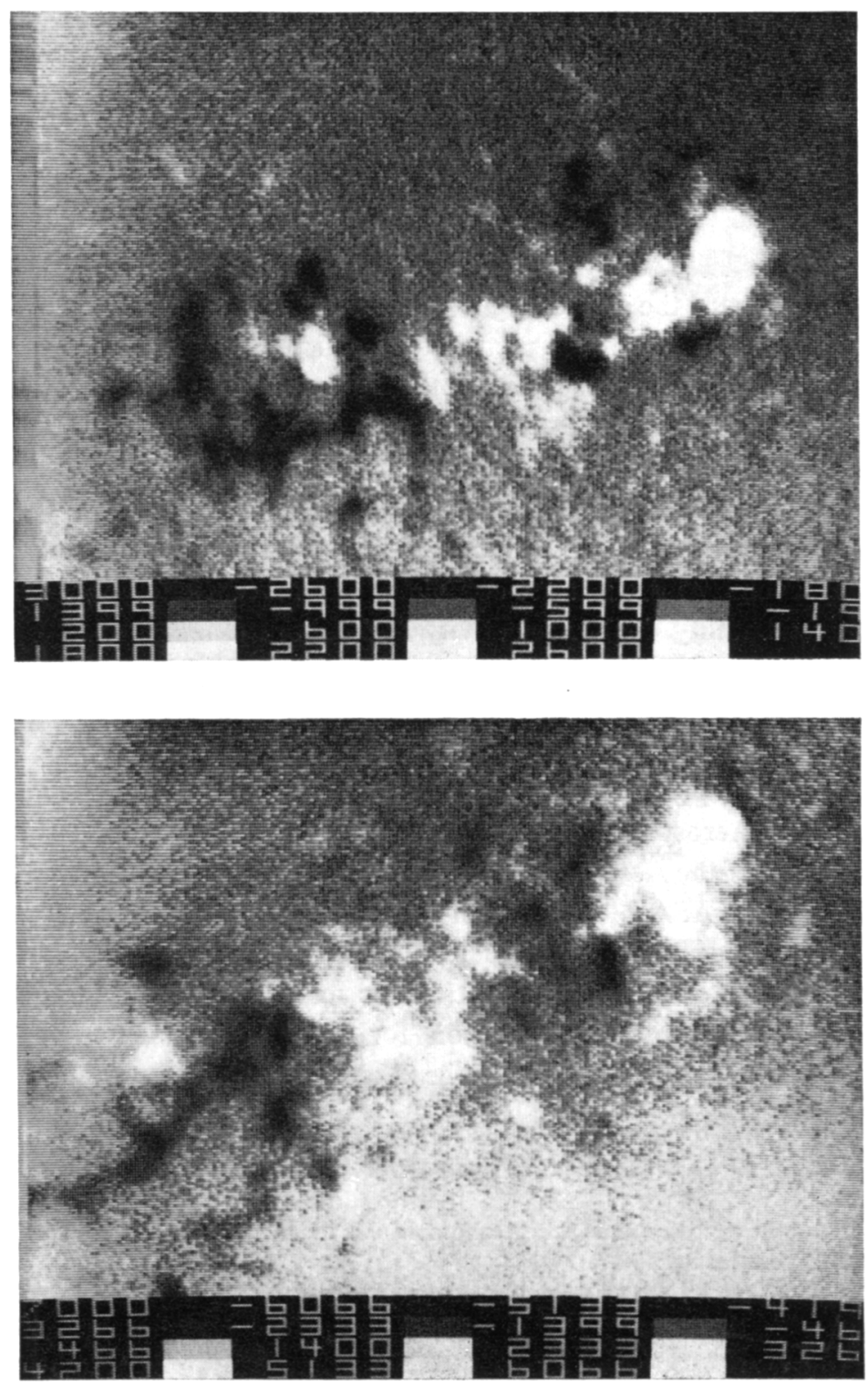

Fig. 4. Videomagnetograms showing the development of an active region during three days from October 19 to 22, 1970. The numbers next to the density bars are not calibrated in Gauss. 
The display section can also be used for graphic and alphanumeric display of the data, ranging from intensity plots on arbitrary cross sections of the picture, to distribution plots of the entire frame.

The analytic section consists of a Varian $620 \mathrm{i}$ computer and forty-eight tracks on the disc.

This data storage area is used as an intermediate storage to free the data gathering area. From this area, the data can be transferred to permanent storage on tape. It can also be used to hold image correction data which the computer could use to correct the raw data for imperfections or shading in the TV camera. The Varian computer controls the system and is available for further analysis of the data either in real time or later. The digital equipment is shown in Figure 2.

\section{Results and Present Status}

The initial results from the Videomagnetograph are shown in Figure 3. The videomagnetogram shown is a polaroid picture of the display monitor. Several days later the magnetic fields from photographic subtraction were available for confirmation of the fields and their polarities.

The initial result is very noisy. Later and somewhat less noisy magnetograms made later do not have matching photographic magnetograms for comparison. Improvements in the video preamplifier is expected to greatly improve the sensitivity but this and other improvements are not yet complete. The present effort is mostly directed toward greater reliability, higher sensitivity and calibration.

\section{Acknowledgements}

The authors wish to express their appreciation to E. B. Mayfield, G. A. Paulikas, and R. A. Becker of the Aerospace Corporation for their continued support of this project, to Greg Kozlowski of the Aerospace Corporation for technical support in all areas, to Donald Robbins of NASA MSFC for his continual collaboration, to Dave Rutland of Spatial Data for design and construction of the high speed digital electronics, to Dale Vrabec of the Aerospace Corporation for many helpful discussions, to Gary Chapman of the Aerospace Corporation for taking simultaneous photographic magnetograms and to Mary Gates of the Aerospace Corporation for her aid in assembly of reports.

\section{Discussion}

Deubner: What was the exposure time for the photographic magnetogram, as compared with that for the video-magnetogram taking $40 \mathrm{~s}$ ?

Janssens: The scan time for the photographic magnetic exposure was two minutes. 